\title{
A Review of Additive Manufacturing Technologies
}

\author{
Cosmina Chiujdea ${ }^{1}$, Sorin Cananau ${ }^{1}$ \\ ${ }^{1}$ Doctoral School of Industrial Engineering and Robotics Faculty, University Politehnica of \\ Bucharest, Romania \\ E-mail: q.cosmina@yahoo.com
}

\begin{abstract}
Over time, companies have prioritized the management of money, materials, equipment and people. Today, in such a globalized environment, they have come to recognize the paramount importance of quality, price, fast product delivery, flexibility and a continuous process of innovation. This is where additive manufacturing comes into play as one of the newest and most promising improvement solution for the above mentioned components of the industry. This paper will provide an understanding of the additive manufacturing technologies, analize them in comparison to the traditional machining techniques, emphasizing their application fields and their limitations and will present the various types of said technologies. There is still a lot of work and research to be done until additive manufacturing technologies overtake the manufacturing world but the continuous growth and successful results that have been seen since the beggining show the great undeniable potential that these technologies have.

Keywords: additive manufacturing, computer-aided design, three-dimentional model..
\end{abstract}

\section{Introduction}

Additive manufacturing ( $\mathrm{AM}$ ), technologies which are fundamentally different from traditional substractive manufacturing techniques, in the sense that they use another principle for the materialization of a part, are technologies that allow the part to be created by adding material, as much as necessary and where is necessary. With these new technologies a physical product, a prototype or a final part is made, layer by layer, starting from a 3D model.

In comparison with subtractive manufacturing processes, in which one starts with a block of material and removes any unwanted material until the desired part is left, additive manufacturing starts with nothing and builds the part one layer at a time by adding each new layer on top of the previous one, until the part is complete.[1] Each layer is a thin cross-section of the part derived from the original CAD data (figure 1). Every layer must have a finite thickness to it and so the thinner each layer is, the closer the final part will be to the original. All commercialized AM machines to date use a layer-based approach and the major ways that they differ are in the materials that can be used, how the layers are created, and how the layers are bonded to each other. Such differences will determine factors like the accuracy of the final part plus its material properties and mechanical properties. They will also determine factors like how quickly the part can be made, how much post-processing is required, the size of the AM machine used, and the overall cost of the machine and process.[2] 

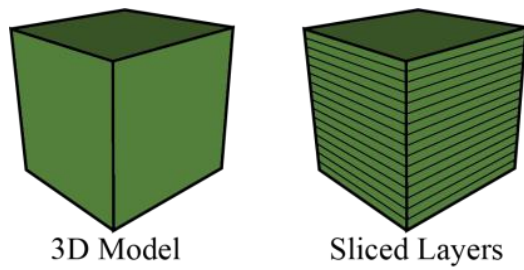

Figure 1. Slicing of a part into layers.

\subsection{The additive manufacturing process chain}

AM involves a number of steps that move from the virtual CAD model to the physical resultant part. The additive manufacturing process chain is shown in figure 2 .

$$
\text { 3D CAD modeling } \rightarrow \text { Conversion to STL } \rightarrow \text { Building } \rightarrow \text { Remova } \rightarrow \text { Post-processing }
$$

Figure 2. The additive manufacturing process chain.

The first step in the additive manufacturing process is always the creation of the digital model. There is a vast variety of $3 \mathrm{D}$ computer aided design (CAD) software that can be used to obtain a fully enclosed volume. There are other methods to obtain the desired model too, such as reverse engineering or tomography, but these are not as flexible as the first mentioned.

The next step is the conversion of the CAD model to an STL file (STereoLithography). STL has several backronyms such as "Standard Triangle Language" and "Standard Tessellation Language". Most CAD system can output an STL file, which is used as the basis of layering the model. STL is a simple way of describing the shape of the model by approximating the model surface with triangular facets.[4] After an STL file has been generated, the file is imported into a slicer program. This program takes the STL file and converts it to G code. G code is a numerical control (NC) programming language. It is used in computer aided manufacturing (CAM) to control automatic machine tools (including CNC machines and $3 \mathrm{D}$ printers). The slicing program allows the designer to customize the construction parameters, including the support, the layer height and the orientation of the part.

Once the software has sent the part build instructions to the machine, it starts to build the part layer upon layer. There are several ways to form a cross section, depending on the particular technology being used. Some AM machines use computer-controlled scan head (laser scanner, nozzle, cutting knife, etc.) to scan lines in order to form a layer cross section (through curing resin, sintering powder, binding powder, cutting paper, etc.). The newly formed layer will be bonded to the previous one until the whole part is finished.[4]

After the part is finished being built, it has to be removed from the machine and post-processed. The removal requires interaction with the machine, thus safety measures must be taken. Post-processing has to be done next, with extreme caution, since the parts might be weak at this point, or they still have support material in need of removal. Cleaning the part of left-over powder or resin might also be required, and, in many cases, even further processing such as machining, if a surface requires a finer finish than the AM machine can provide, infiltration to make the part stronger, heat treatment for metal parts, or colouring and painting if the part needs to be in a colour other than that provided by the AM material.[1]

\subsection{Current Usage of Additive Manufacturing}

In recent years, AM technologies have been increasingly applied in various industry sectors to improve the material performance and enhance energy efficiency and have been considered as one of the nextgeneration solutions coming with advantages such as cost reduction, speed, accessibility, sustainability, ability to create complex geometries. [5]

Wohlers Associates is a company that helps define, coordinate, and review the results of experiments that test methods of rapid product development, additive manufacturing, and 3D scanning/imaging. 
According to their 2018 annual 'state of the industry' report [6] the areas of use for AM are as shown in figure 3 .

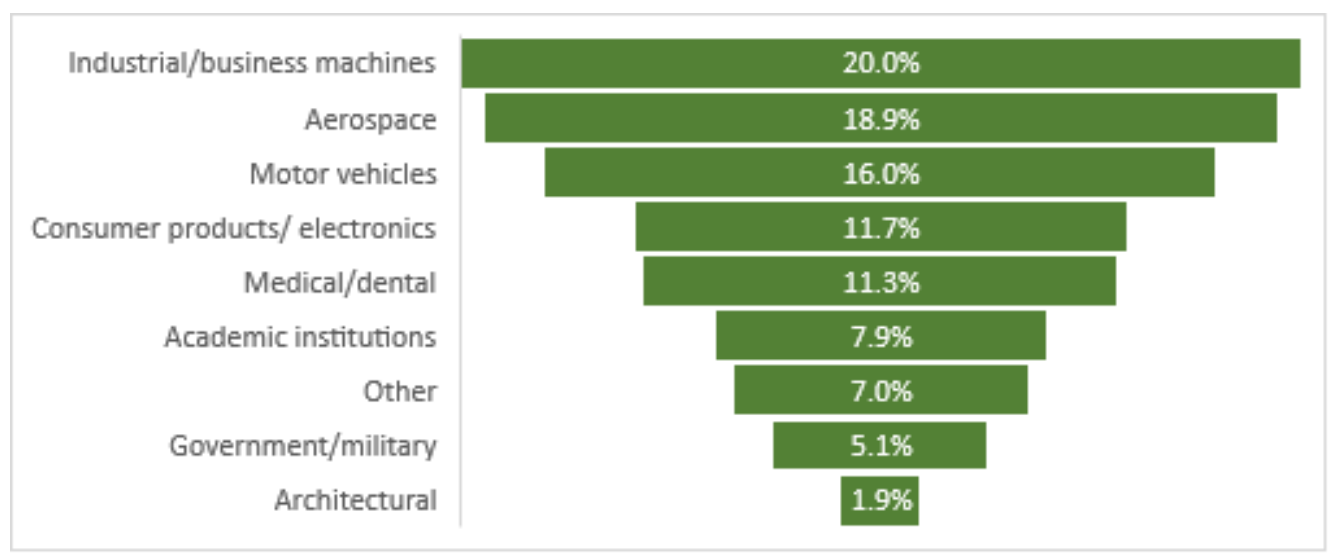

Figure 3. Industries using additive manufacturing, according to the 2018 Wholers Report.

Although there are many advantages in choosing these technologies, as they have been scarcely pointed out in this paper thus far, AM also comes with its disadvantages. These include the relatively high cost of the machines and operation, slow production rate, limited materials and the shrinkage of fabricated parts.

\section{Types of additive manufacturing technologies}

There are many additive manufacturing processes available and there has yet to be determined an ideal classification of all of them. What makes it difficult to do so are the their differences in terms of materials, energy sources, types of feedstocks used and conveyance of feedstocks. The struggle is to find a classification that will not exclude any of the processes while also allow room to accommodate new ones that have not been discovered yet.[3]

Although the classification of AM processes is an ongoing debate, with new process techniques emerging every day, this present paper will only introduce the categories published by the American Society for Testing and Materials (ASTM) Committee F42 on Additive Manufacturing Technologies (Standard Terminology for Additive Manufacturing Technologies, 2012), which, broadly, divides them based on how the material is solidified, as follows: Vat Photopolymerization, Material Jetting, Binder Jetting, Material Extrusion, Powder Bed Fusion, Sheet Lamination and Directed Energy Deposition.[7]

\subsection{Vat Photopolymerization}

Vat Polymerisation technologies uses a vat of liquid photopolymer resin, out of which the model is constructed layer by layer. An ultraviolet (UV) light is used to cure or harden the resin where required, whilst a platform moves the object being made downwards after each new layer is cured. The main technologies in this category are stereolithography (SLA) using either lasers or DLPs (Digital Light Processing), and Continuous Liquid Interface Production (CLIP).[7] Out of these three, Stereolithography is the first version of additive technology invented and is the most widely used AM process technique nowadays.

As it can be seen in figure 4 the platform starts at one layer's thickness beneath the surface of the polymer. The computer uses the sliced model information to control the dynamic mirrors, which direct the laser beam over the vat surface, scanning the cross section of one layer of the part. The platform then descends into the resin, allowing a fresh thin film to form over the previous one. The laser starts scanning the new layer, building the next slice as well as bonding it to the previous one. The process is repeated until the desired part is built. The waste-free quality of this process is highlighted by the fact 
that the leftover resin can be used for a future builds. For overhanging features of the part, easily removable synthetic supports can be designed to ensure the correct formation.[1][4]

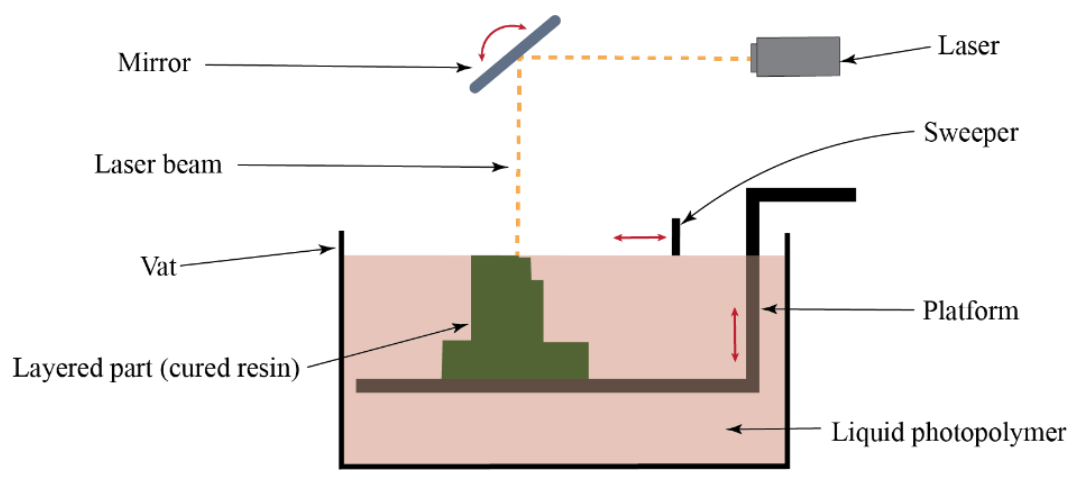

Figure 4. Schematic representation of the Stereolithography process.

\subsection{Material Jetting}

Material jetting creates objects in a similar manner to an inkjet printer. Material is jetted onto a build platform using either a continuous or Drop on Demand (DOD) approach.

The printer head moves horizontally above the platform, depositing liquid photopolymer material onto it for each layer of the model. The material is then cured by UV light or allowed to cool and harden. Machines vary in complexity and in their methods of controlling the deposition of material. The material layers are then cured or hardened using ultraviolet light. The process is then repeated until the completion of the part. In figure 5 it can be seen that the print-head is designed to deposit both the part material, as well as any required support material that will subsequently need to be removed.

As material must be deposited in drops, the number of materials available to use is limited. Polymers and waxes are suitable and commonly used materials, due to their viscous nature and ability to form drops.[7]

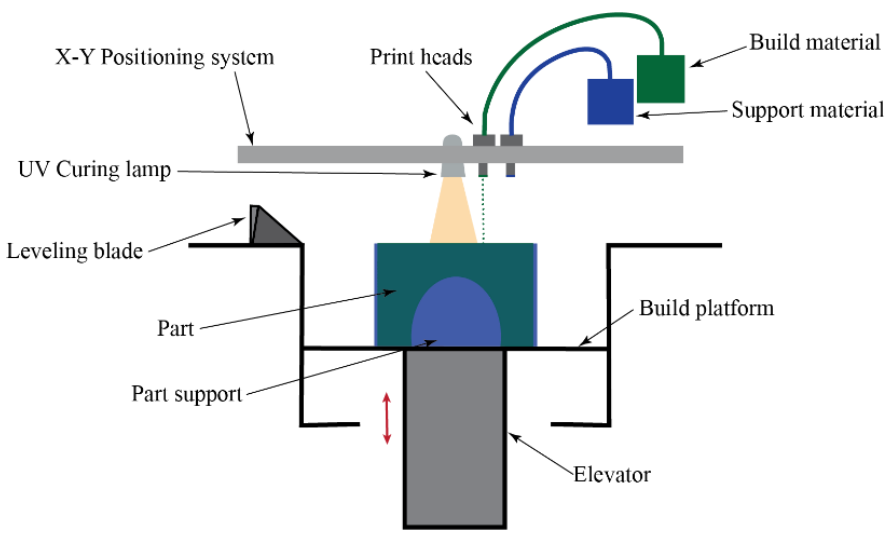

Figure 5. Schematic representation of the Material Jetting process.

\subsection{Binder Jetting}

Similar to the technologies presented so far, the Binder Jetting process makes use of a descending platform, building the product layer by layer. In this case the materials used in the process are a powder based material that is spread over the build platform using a roller, and a binder, usually liquid, that is deposited on top of the powder where necessary. The print head moves horizontally depositing alternating layers of the build material and the binding material thus creating the final piece. Figure 6 shows the schematic representation of the Binder Jetting process. 
In contrast to the others, this process does not require additional supports as the unbound powder serves this role, being easily removed after completion.

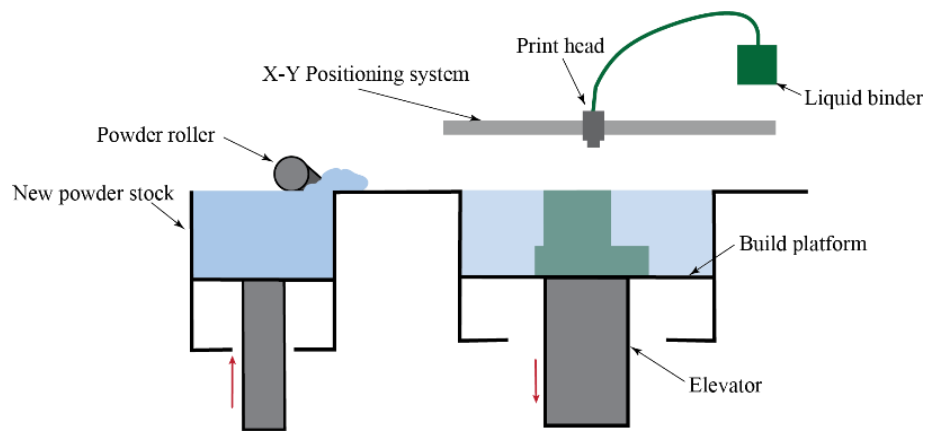

Figure 6. Schematic representation of the Binder Jetting process.

\subsection{Material Extrusion}

The Fuse deposition modelling (FDM) is shown in figure 7. This is one of the most common and accessible additive manufacturing technologies. Material is being fed to a nozzle, where it is heated and then deposited in a continuous stream onto the moving platform. Each new layer fuses automatically to the previous because of its melted state.

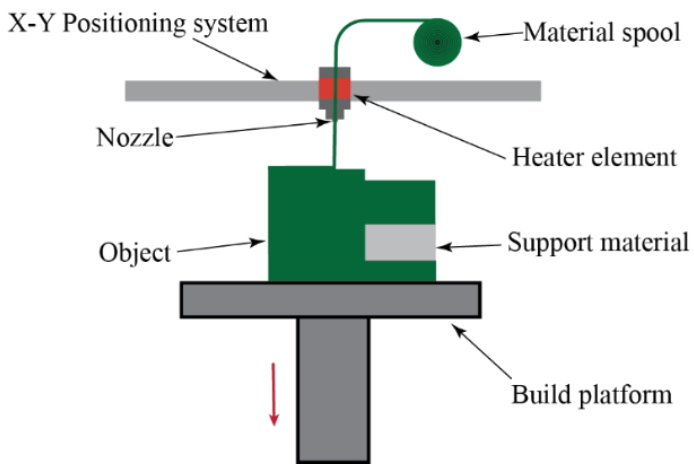

Figure 7. Schematic representation of the Material Extrusion process.

\subsection{Powder Bed Fusion}

Powder Bed Fusion technologies includes: Direct metal laser sintering (DMLS), Electron beam melting (EBM), Selective heat sintering (SHS), Selective laser melting (SLM) and Selective laser sintering (SLS).

The Powder Bed Fusion process, shown in figure 8, makes use of a moving platform on which the build material, in powder form, is spread. This method uses a laser or an electron beam to both melt the current layer and, at the same time, bond it to the previous one. Wherever the fusing agent has been printed, the powder absorbs enough of the heat energy to melt, whereas the rest of the material remains in unfused powder form. These technologies can produce parts in both a variety of polymers (in the case of LS) and metals (in the case of SLM and EBM).[1] 


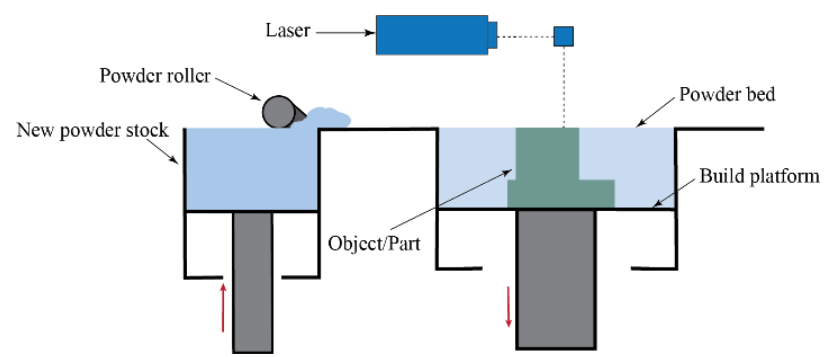

Figure 8. Schematic representation of the Powder Bed Fusion process.

\subsection{Sheet Lamination}

Sheet lamination processes include laminated object manufacturing (LOM) and ultrasonic additive manufacturing (UAM).

LOM involves layer-by-layer lamination of paper material sheets, cut individually, each sheet representing one layer of the CAD model. In LOM, the excess portions of the paper sheet is sliced into cubes using a cross-hatch cutting operation for easy removal post build, as it can be seen in figure 9.[2]

The UAM process uses sheets or ribbons of metal. The material is cut into shape using a laser and then bound together with the previous layer using ultrasonic welding. These two steps are interchangeable as the material can be bonded before being cut. The process does require additional $\mathrm{CNC}$ machining and removal of the unbound metal, often during the welding process.[7]

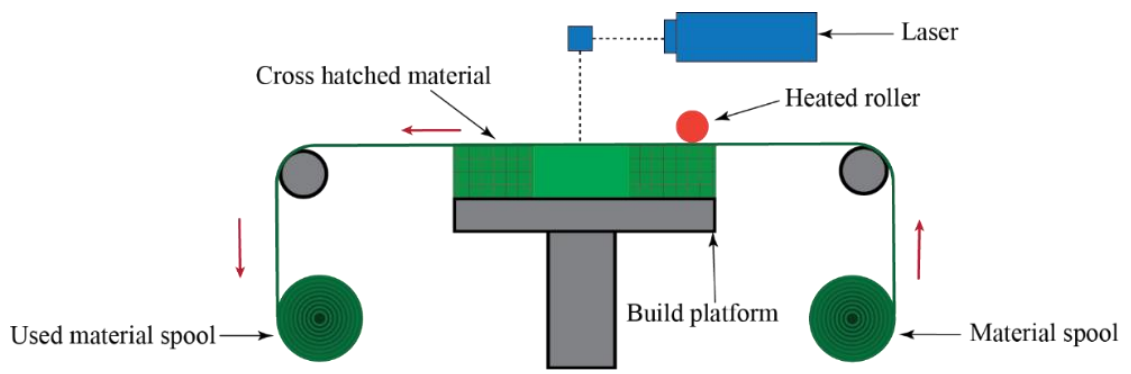

Figure 9. Schematic representation of the Sheet Lamination process.

\subsection{Directed Energy Deposition}

Directed Energy Deposition (DED) covers a range of technologies including LENS (Laser engineered net shaping), DMD (direct metal deposition), CLAD (3D laser cladding). It is a process commonly used to repair or add additional material to existing components.

A DED machine functions on the same principle as material extrusion, with the difference that the nozzle is fixed on a 4 or 5 axis arm that moves around a fixed object. Figure 10 shows how the material, which can be deposited from any angle, is melted upon deposition with a laser or electron beam. The process can be used with polymers, ceramics but is typically used with metals, in the form of either powder or wire.[7]

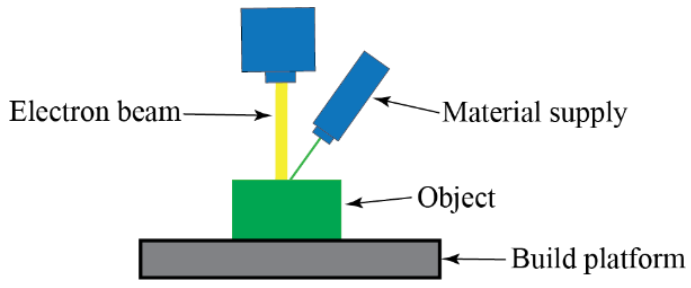

Figure 10. Schematic representation of the Directed Energy Deposition process. 


\section{Surface quality in additive manufacturing}

Additive Manufacturing technologies have progressed in the past few years and are even capable of producing functional parts instead of just prototypes, but so far it has some drawbacks. One of the main issues, that needs significant improvement, is the surface texture and integrity. The surface quality is very important, as it can influence the accuracy and performance of the product, and minimize the quantity of post-processing operations. The surface roughness of the produced surface has been the main concern of many studies focused on determining and controlling the different factors affecting it.

Sikder et al.[8] proposes a new adaptive algorithm which globally optimizes a texture error function produced by the staircase effect for a user-defined number of layers. The adaptive slicing algorithm dynamically calculates optimized slicing thicknesses based on the rapid prototyping machine's specifications to minimize the texture error function. By comparing their results with the common practice for several other case studies, they showed that utilizing this approach can lead to the number of prototyping layers to be reduced by 20-50, while maintaining or improving the accuracy of the final manufactured surfaces. Anitha et al.[9] used the Taguchi statistical approach in the attempt to obtain the optimum process conditions for a minimised surface roughness on FDM prototypes. The parameters they studied are layer thickness, road width and speed of material deposition on the surface roughness. They used three levels for each parameter and studied the effect of each one of them on the final surface roughness. Bacchewar et al.[10] studied the effect of build orientation, laser power, layer thickness, beam speed, and hatch spacing, on surface roughness of SLS parts. They used central rotatable composite design (CCD) to plan the experimental work and analysis of variance (ANOVA) to study the significance of process variables on the surface roughness. They found that in the case of upward-facing surfaces (surface angle less than 90 degrees), build orientation and layer thickness have been the significant parameters. In downward facing surfaces (surface angle between 90 and 180 degrees), other than build orientation and layer thickness, laser power has also been found to be significant.

Safdar et al.[11] developed a mathematical model based upon response surface methodology (RSM) in order to study the variation of surface roughness with changing process parameter settings on EBM fabricated Ti-6Al-4V metallic parts. They studied the surface roughness of the test slabs produced with different parameter settings and thickness under confocal microscope, revealing that the surface roughness parameter $R_{a}$ varies between 1-20 mm in each different case. It was found that the $R_{a}$ value increases with increasing sample thickness and beam current, and decreases with the increase in offset focus and scan speed.

Ahn et al.[12] present a methodology to quantify the surface roughness of parts processed by Laminated Object Manufacturing (LOM). They studied the surface profiles of the parts by surface angle variation, and constructed a schematic that can express surface profiles by considering the LOM process factor geometrically. A theoretical model to quantify the average surface roughness was proposed, and the expressions required for numerical computation were deduced and defined. The proposed approach was verified by comparing measured data with computed values. Additionally, the effects of the process variables related to surface quality, such as surface angle, layer thickness, cutting shape, and penetration depth, were analyzed and evaluated. Their method can be used to predict the surface roughness of AM fabricated millimeter structures or micro parts.

When using powder-bed fusion, the part is being built by melting metal-powder particles with a laser, a process that leaves rough surfaces, with partially melted powder particles potentially being stuck to the sides of the part. Rombouts et al.[13] studies the effects of surface inclination angle and strategies to improve the surface finish of Laser Metal Deposition (LMD) components. The experiment they conduct show that by laser remelting after powder deposition a substantial improvement in surface quality of both the side and top surfaces can be obtained.

An industry-related survey [14] inffered that most AM parts require post-processing (computer numerical control machining, abrasive machining, laser machining, chemical treatments, etc.) to fulfill the minimum surface roughness levels required by the industry.

To conclude this chapter, it is important to point out that the surface quality, an imperial factor for the functionality of most parts, can be affected by various different factors in the AM process. These 
factors are present in all production stages, from pre-processing, processing to post-processing, varying from one AM machine to another.

\section{Conclusions}

In conclusion, this paper shows that additive manufacturing benefits from multiple and important advantages. These advantages allow the successful use of AM in many different fields.

Like any new technology though, AM still has limitations and disadvantages that still require quite a lot of experimental research. The low number of usable materials, surface quality issues and accuracy levels, tolerances, and the limited build chamber volumes are major technical barriers that need to be overcome in order to achieve the large-scale industrial use of AM technologies. Nevertheless, even if AM will not eliminate conventional manufacturing technologies any time soon, it will most definitely operate side by side with them from now on.

The present study gives a small insight in the world of AM technologies, the multitude of different processes there are and the effect of process parameters on the final product. Since materials play a dominant role in additive manufacturing, for the future, a more detailed study regarding how these influence the surface quality of the built part is desired. An engineer could make use of such a study in order to make the optimal choices well in advance of the manufacturing the part.

\section{References}

[1] Olaf Diegel, Axel Nordin, Damien Motte. "A Practical Guide to Design for Additive Manufacturing". Springer Series in Advanced Manufacturing, 2019.

[2] Brent Stucker, David Rosen, Ian Gibson. "Additive Manufacturing Technologies: 3D Printing, Rapid Prototyping, and Direct Digital Manufacturing”. Springer, 2010.

[3] Sanjay Kumar. "Additive Manufacturing Processes". Springer, 2020.

[4] Lian Q., Xiangquan W., Dichen L. "Additive Manufacturing Technology”. Digital Orthopedics. Springer, 2018

[5] Cheng Sun, Yun Wang, Michael D. McMurtrey, Nathan D. Jerred, Frank Liou, Ju Li."Additive manufacturing for energy: A review". Nano energy, ScienceDirect, 2010.

[6] Wohlers Report 2018: 3D printing and additive manufacturing state of the industry. 2018. ISBN 978-0-9913332-3-3

[7] The Additive Manufacturing Reasearch Group (AMRG) at Loughborough University. "About Additive Manufacturing". Loughborough University, 2021. viewed 15 January 2021, $<$ https://www.lboro.ac.uk/research/amrg/about/the7categoriesofadditivemanufacturing/>.

[8] Sikder S., Barari A., \& Kishawy H. A. "Global adaptive slicing of NURBS based sculptured surface for minimum texture error in rapid prototyping". Rapid Prototyping Journal, 2015

[9] Anitha R., Arunachalam S., \& Radhakrishnan P. "Critical parameters influencing the quality of prototypes in fused deposition modelling". Journal of Materials Processing Technology, 2001.

[10] Bacchewar P. B., Singhal S. K., \& Pandey P. M. "Statistical modelling and optimization of surface roughness in the selective laser sintering process". Proceedings of the Institution of Mechanical Engineers, Part B: Journal of Engineering Manufacture, 2007.

[11] Safdar A., He H. Z., Wei L., Snis A. \& Chavez de Paz L. E. " Effect of process parameters settings and thickness on surface roughness of EBM produced Ti-6Al-4V". Rapid Prototyping Journal, 2012.

[12] Ahn D., Kweon J.-H., Choi J., \& Lee S. "Quantification of surface roughness of parts processed by laminated object manufacturing". Journal of Materials Processing Technology, 2012.

[13] Rombouts M., Maes G., Hendrix W., Delarbre E., \& Motmans F."Surface Finish after Laser Metal Deposition”. Physics Procedia, 2013.

[14] Kretzschmar N.; Chekurov S.; Salmi M.; Tuomi J. "Evaluating the readiness level of additivelymanufactured digital spare parts: an industrial perspective". Appl. Sci, 2018. 